\title{
Hypothesis: when is a seminoma not a seminoma?
}

\author{
D RAGHAVAN, * EADIE HEYDERMAN, *§ P MONAGHAN, * J GIBBS, $†$ \\ ERUOSLAHTI,$\ddagger$ MJ PECKHAM $†$ AND AM NEVILLE*
}

From * the Unit of Human Cancer Biology, Ludwig Institute for Cancer Research, Royal Marsden Hospital, Sutton, Surrey SM2 SPX; †the Institute of Cancer Research and Royal Marsden Hospital, Sutton, Surrey SM2 5PT; and $₫$ La Jolla Cancer Research Foundation, La Jolla, California, USA 92038

SUMmaRY A xenograft line, HX 53, has been established in immune-suppressed mice from a specimen of a lymph node metastasis in a patient with a histological diagnosis of seminoma but with markedly raised circulating levels of alpha-fetoprotein. Histological, immunocytochemical, and ultrastructural studies of this xenograft line have suggested that a solid variant of yolk sac carcinoma may exist, which morphologically resembles seminoma, or that a continuum of differentiation exists between seminoma and yolk sac carcinoma.

The histogenesis and classification of seminoma and malignant teratoma have been subjects of controversy for many years. ${ }^{1-4}$ As part of a broad investigative programme, we have established as a xenograft a new human germ cell tumour line, HX 53, which has the light microscopical appearance of seminoma but functional and ultrastructural traits of a form of malignant teratoma, a yolk sac carcinoma.

\section{Methods}

Tissue for xenografting was obtained from a lymph node metastasis from a patient with a histological diagnosis of seminoma but with elevated circulating levels of alpha-fetoprotein (AFP) (see below). Tumour fragments were implanted in thymectomised and irradiated mice as described elsewhere. ${ }^{5}$ Samples of blood and tumour cyst fluid were assayed for AFP by radioimmunoassay. Indirect immunocytochemical staining for AFP and human chorionic gonadotrophin (HCG) were performed according to the technique of Heyderman and Neville ${ }^{7}$ with affinitypurified anti-AFP8 and antisera raised to the $\beta$-subunit of HCG (Hoffman-La Roche). Negative controls were established by ensuring complete extinction of staining when antisera were absorbed with purified AFP or HCG before use. All xenografted tissues for routine histological and immunocyto-

§Present address: Department of Morbid Anatomy, St Thomas' Hospital Medical School, London SE1 7EH.

Accepted for publication 27 August 1980 chemical staining were fixed in Bouin's reagent.

\section{Case history}

A 37-year-old white man presented with a ninemonth history of left testicular enlargement. Orchidectomy was performed. The histological diagnosis was seminoma; tumour markers were not assayed at that time. A postoperative CT-scan demonstrated para-aortic node involvement, and the patient underwent irradiation. Subsequent CT-scans showed a persistent abnormality in the abdominal lymph nodes, and the patient was referred to the Royal Marsden Hospital with advanced abdominal disease and cervical lymphadenopathy. Serum AFP at this time was $6600 \mu \mathrm{g} / \mathrm{l}$. A node biopsy, the source of xenografted material, revealed active malignant disease (see below). The patient was treated with combination chemotherapy (vinblastine $0.3 \mathrm{mg} / \mathrm{kg} \mathrm{IV}$ days 1 and 2, bleomycin 30 U IV days 1-5, every month) and achieved partial remission with decreasing abdominal disease and falling AFP. However, AFP titres began to rise after the third course, accompanied by widening of the mediastinal shadow on chest $x$-ray. Therapy was altered to VP 16-213, $200 \mathrm{mg} \mathrm{IV} \times 5$, and cis-platinum, $100 \mathrm{mg} / \mathrm{m}^{2}$ IV stat, and again a transient partial response was achieved. Palliative mediastinal irradiation was begun but with little effect. Repeat CT-scans in August demonstrated increasing abdominal tumour and liver secondaries. The patient died in September 1978, 21 months after initial presentation. Permission for necropsy was not given. 


\section{Results}

CONVENTIONAL HISTOLOGY

Histological examination of the primary testicular lesion revealed a tumour composed of sheets of uniform, clear, polygonal cells with distinct cell membranes, finely granular yet open cytoplasm, and prominent vesicular nuclei (Fig. 1). Few mitoses were seen. There was scanty lymphocytic infiltration, but granulomata were numerous, and there were areas of necrosis. No tumour giant cells were seen. The diagnosis of seminoma was confirmed by several histopathologists. Re-examination of the material after the xenograft data became known (see below) showed the presence of small, extracellular, eosinophilic, PAS-positive globules often related to minute cystic spaces surrounded by a few tumour cells.

The histology of the node metastasis was similar to that of the primary tumour but with more prominent areas of necrosis and increased nuclear pleomorphism and mitotic activity. Again, extracellular, eosinophilic globules were noted in association with small cystic spaces which were more prominent than in the primary tumour. Lymphocytic infiltration was sparse, and no giant cells were seen.

The fragments implanted into the mice developed to form a distinct tumour which is referred to as the HX 53 line. Specimens from serial xenograft trans- plants up to 10 passages showed retention of the basic tumour pattern and consisted of solid sheets or trabeculae of tumour cells which had well-defined cell membranes, open clear cytoplasm, and single rounded vesicular nuclei with prominent nucleoli. Nuclear pleomorphism and mitotic activity were marked. The appearances are consistent with those referred to as 'anaplastic seminoma'. In association with this pattern were areas of microcyst formation, each being surrounded by tumour cells similar to those of the solid areas of the lesion (Fig. 2). Such areas of cyst formation together with the microcystic lacy pattern are characteristic of endodermal sinus tumour (yolk sac carcinoma). The occasional Schiller-Duval body was also present. These two patterns were intimately interspersed and admixed one with another.

\section{DEMONSTRATION OF AFP}

AFP was demonstrated by radioimmunoassay in the blood of mice bearing the xenografts and by immunoperoxidase in the cytoplasm of the tumour cells surrounding the cystic areas and in the cysts themselves. Granular cytoplasmic staining for AFP was also noted in some cells of the solid 'seminomatous' regions, particularly in areas bordering the cysts (Fig. 3). We have shown previously that formalin may vitiate the demonstration of AFP immunocytochemically; the primary and metastatic tumours

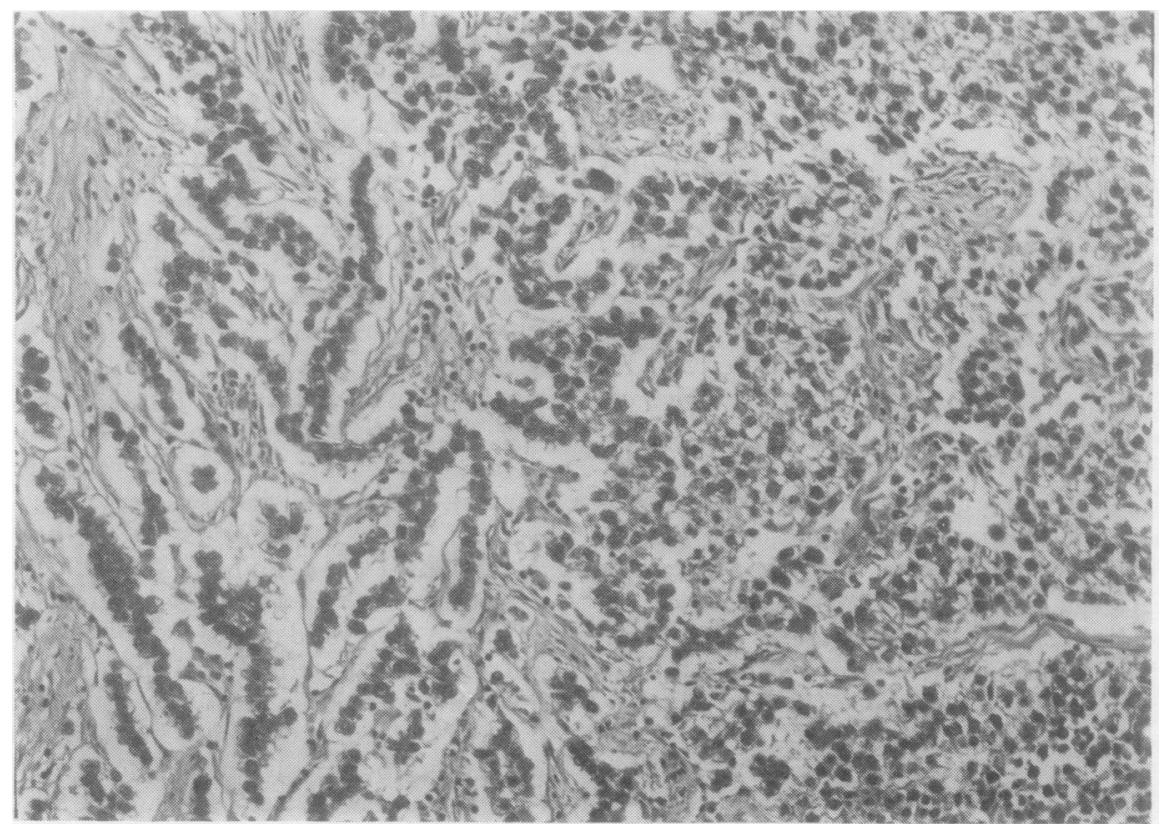

Fig. 1 Primary tumour of testis. The classical seminoma pattern is shown on the left with a solid sheet of polygonal cells on the right. (Haematoxylin and eosin $\times 160$ ) 


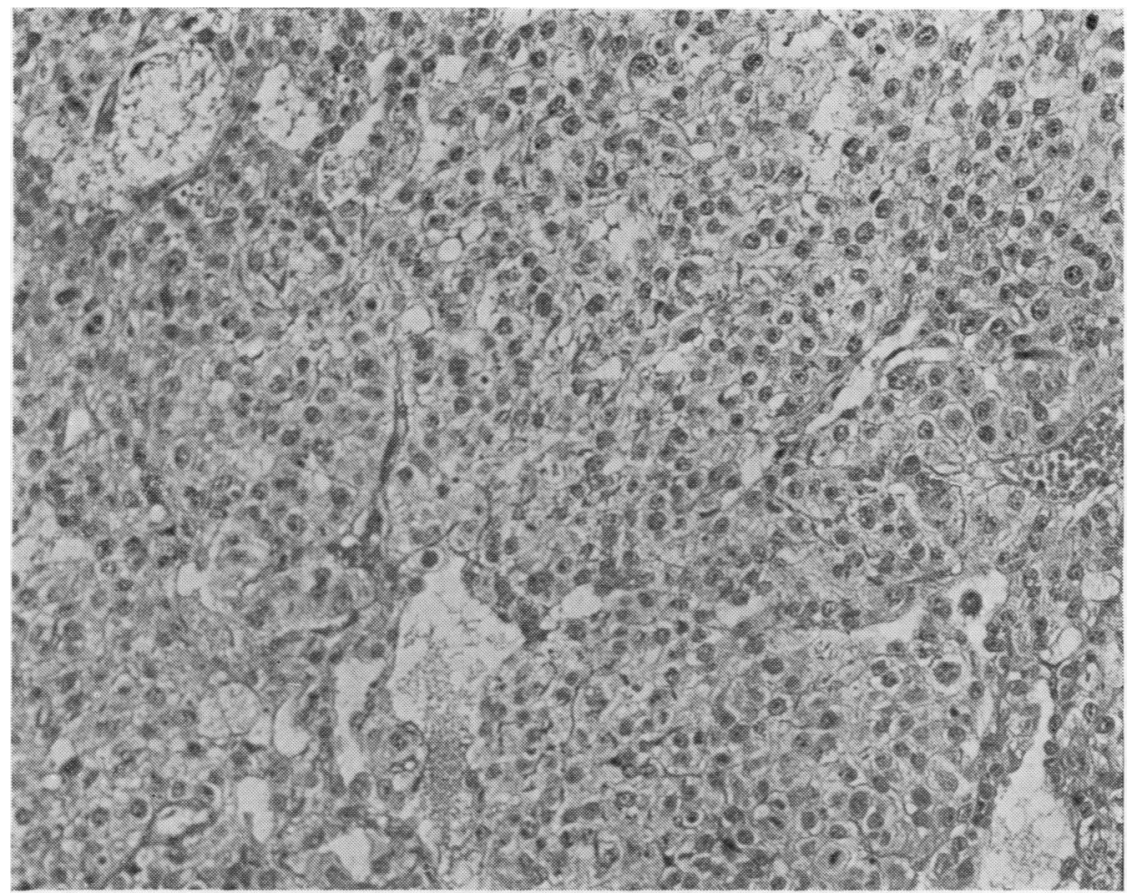

Fig. 2 Xenograft tumour. The lesion retains the pattern of solid sheets of cells but also reveals foci of microcyst formation. ( $H$ and $E \times 200$ )

were fixed in formalin, and attempts at tissue staining for AFP were uninterpretable due to variability of the pattern and apparent 'background' staining. HCG staining was negative in all specimens examined.
ULTRASTRUCTURE

In the cystic areas, the tumour cells had the ultrastructural features of yolk sac carcinoma (numerous cytoplasmic organelles, profuse rough endoplasmic reticulum, microvilli, many desmosomes in inter-

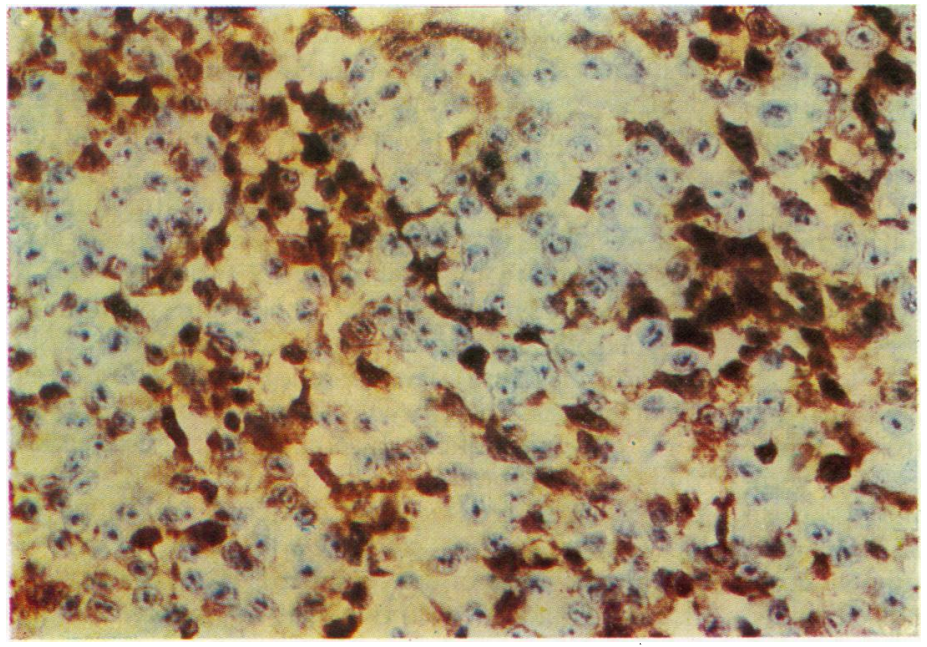

Fig. 3 Xenograft tumour. The dark area represents immunoperoxidase staining of alpha-fetoprotein. $(\times 250)$ 


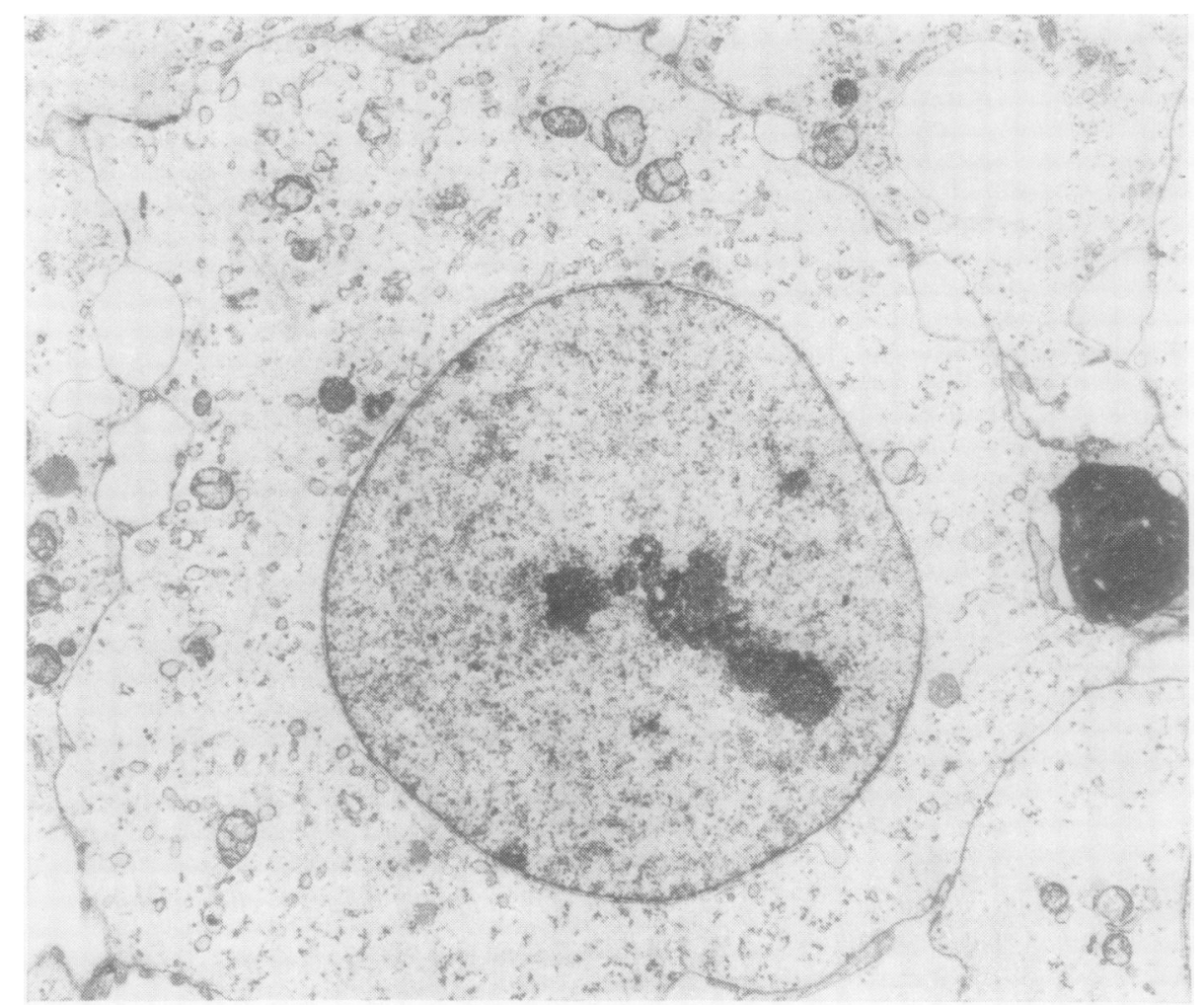

Fig. 4 Xenograft tumour. A representative tumour cell of the intermediate type showing a round nucleus, prominent nucleolus, scattered desmosomes, intercellular microcysts, and osmiophilic material. $(\times 6500)$.

digitating cell membranes, tight junctions in relation to the cysts, and extracellular osmiophilic deposits). In the solid areas, the electron microscopical appearances were of 'classical' seminoma cells (empty cytoplasm, glycogen, no desmosomes, no microvilli, and no extracellular osmiophilic deposits). Between the solid and cystic areas of the tumour, cells with intermediate ultrastructural features could be found together with both types of cell described above (Fig. 4). The original formalin-fixed lymph node biopsy specimen was reprocessed for electron microscopy. Despite the poor preservation, extracellular osmiophilic deposits were readily observed.

\section{Discussion}

Although it is now widely accepted that seminomas and teratomas are derived from undifferentiated germ cells, ${ }^{1-4}$ the developmental relationship between seminoma and malignant teratoma remains unclear, as does the histogenesis of the 'mixed tumour' composed of seminomatous and teratomatous elements.
Based on studies of mixed tumours in which the seminomatous elements consisted of distinct foci, it has been postulated that their derivation is distinct from the non-seminomatous components. ${ }^{49}$

The problems of differentiating between the development of seminoma and teratoma have been increased by the marked similarities in the light and electron microscopical features of 'undifferentiated seminoma', ${ }^{10}$ anaplastic seminoma, ${ }^{211}$ and embryonal cell carcinoma. ${ }^{10}$ The major ultrastructural features that separate the non-seminomatous germ cell tumours from seminomas appear to be their increased nuclear irregularity, their more pronounced cytoplasmic differentiation, the greater frequency of desmosomes and tight junctions, the presence of cystic spaces, and (in yolk sac carcinomas) the presence of osmiophilic extracellular structures. Features that may be common to both include rope-like nucleoli, variable glycogen content, and abundant polysomes.

The relevance of the cell line, HX 53, to the classification of germ cell tumours is not yet certain. The 
primary tumour and the metastatic deposit (source of xenograft) fulfil the light microscopic criteria for the diagnosis of seminoma or even possibly anaplastic seminoma. However, the areas of microcyst formation seen in the xenograft were present, in retrospect, even in the original biopsy. These have become more prominent histologically in the xenografts. It is these areas that stain most densely for AFP. The presence of granular cytoplasmic AFP staining in areas of the seminoma-like tissue, however, suggests that this is not merely a 'combined' tumour with seminoma and yolk sac elements.

Furthermore, electron microscopic examination has also revealed populations of tumour cells showing a gradation in the degree of differentiation from cells like classical seminoma to those with features typical of classical yolk sac carcinoma, other cells possessing structural features intermediate between these extremes.

The interpretation that seems to fit the present data best is that, in addition to the well-described cystic form of yolk sac carcinoma, ${ }^{3}$ there exists a yolk sac tumour with a solid cellular pattern which has many of the light microscopical characteristics of seminoma but with ultrastructural and functional characteristics suggesting varying degrees of differentiation along a continuum between classical seminoma and yolk sac tumour. We do not think that there was originally a two-cell population, which has persisted in 10 successive xenograft passages, as this explains neither the presence of granular AFP staining in some of the seminoma-like cells, the admixture of the two types of tumour cell patterns, nor the ultrastructural pattern with features of both cell types. The concept of a continuum of differentiation between seminoma and yolk sac tumour could explain the phenomenon of 'mixed tumours'.

Twenty-five percent of the metastases from seminoma are undifferentiated malignant teratoma (MTU) and $4 \%$ are differentiated teratoma; $;^{2}$ there are reports of patients with primary tumours similar in light morphology to the present primary lesion which have been found subsequently to metastasise as yolk sac carcinoma. ${ }^{3}$ Pure seminoma and yolk sac carcinoma have been reported to occur as a mixed primary lesion, ${ }^{12}$ although the histogenesis was not explained. Up to $10 \%$ of stage I seminomas are not initially cured by orchidectomy and conventional radiotherapy. It may well be that these phenomena are explained in part by inadequacy of diagnosis at the light microscopic level, a solid yolk sac carcinoma or intermediate elements being mistaken for seminoma and treated accordingly. The absence of elevated AFP titres with such tumours does not necessarily preclude the diagnosis of yolk sac carcinoma as it has been well documented that there is marked variability of AFP production by these tumours. ${ }^{13}$

Clinically, the traditional view has been that elevated blood AFP in a patient implies the presence of non-seminomatous elements. In most cases, but not always, rigorous review of histological material has demonstrated teratomatous and/or yolk sac elements when a patient with the histological diagnosis of seminoma has presented with elevated blood AFP levels.

It is essential that marker estimations be performed on all future patients suspected of having germ cell tumours. Moreover, if a more clinically relevant pathological classification of these tumours is to be achieved, it will be necessary to augment routine histological examination with immunocytochemical and possibly ultrastructural studies wherever possible.

We thank Professor Noel Gowing, Dr John Sloane, and Dr Richard Carter for reviewing the pathological specimens, Miss AH Orr for performing AFP estimations, Hoffman-La Roche for their generous gift of anti-HCG antisera, and Diana Mitchell, Pat Davies, Susan Clinton, and John Ellis for meticulous technical assistance.

\section{References}

1 Pugh RCB. Testicular tumours-introduction. In: Pugh RCB, ed. Pathology of the testis. Oxford, London, Edinburgh, Melbourne: Blackwell Scientific Publications, 1976:139-60.

${ }^{2}$ Mostofi FK, Price EB. Tumours of the male genital system. Atlas of tumour pathology. 2nd ser. Fasc 8. Washington DC: Armed Forces Institute of Pathology, 1973.

${ }^{3}$ Teilum G. Special tumors of ovary and testis-Comparative pathology and histological identification. Copenhagen; Munksgaard, 1971.

4 Dixon FJ, Moore RA. Testicular tumors: a clinicopathological study. Cancer $1953 ; 6: 427-54$.

${ }^{5}$ Steel GG, Courtenay VD, Rostom AY. Improved immune suppression techniques for xenografting of human tumours. Br J Cancer 1978;37:224-31.

- Heyderman E, Neville AM. A shorter immunoperoxidase technique for the demonstration of carcinoembryonic antigen and other cell products. J Clin Pathol 1977;30: 138-40.

${ }^{7}$ Heyderman E. Immunoperoxidase technique in histopathology: applications, methods and controls. J Clin Pathol 1979;32:971-8.

${ }^{8}$ Ruoslahti E. Immunochromatography on insolubilized antibodies of very low affinity: application to immunoadsorbence of bovine $a$-fetoprotein. J Immunol 1978;121 : $1687-90$.

9 Pugh RCB. Combined tumours. In: Pugh RCB, ed. Pathology of the testis. Oxford, London, Edinburgh, Melbourne: Blackwell Scientific Publications, 1976; 245-59.

10 Pierce GB Jr. Ultrastructure of human testicular tumors. Cancer 1966;19:1963-83.

11 Janssen M, Johnston WH. Anaplastic seminoma of the 
testis: ultrastructural analysis of three cases. Cancer $1978 ; 41: 538-44$.

12 Talerman A. Yolk sac tumor associated with seminoma of the testis in adults. Cancer $1974 ; 33: 1468-73$.

${ }^{13}$ Grigor KM, Detre SI, Kohn J, Neville AM. Serum alpha ${ }_{1}^{-}$ foetoprotein levels in 153 male patients with germ cell tumours. Br J Cancer 1977;35:52-8.
Requests for reprints to: Dr P Monaghan, Ludwig Institute for Cancer Research, Royal Marsden Hospital, Sutton, Surrey SM2 5PX. 